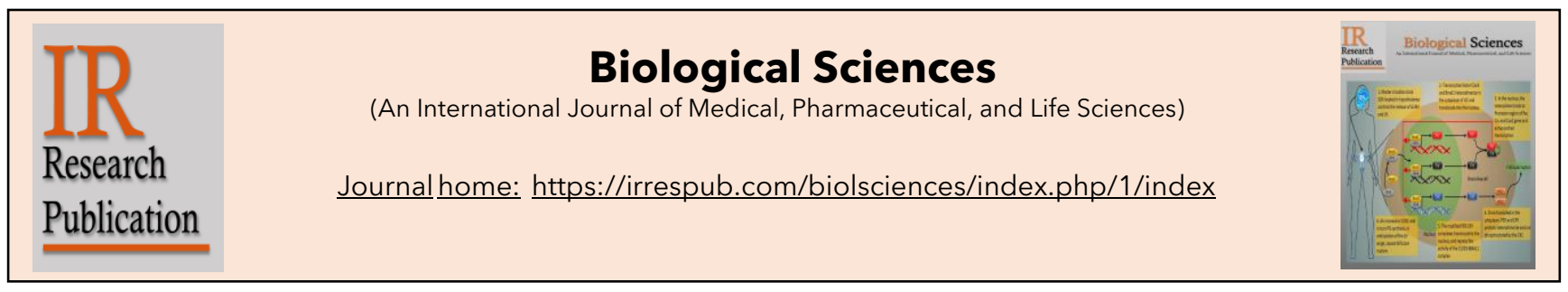

\title{
P53 an oncogene or tumor suppressor gene: A confusion
}

\author{
Jonaid Ahmad Malik* \\ ${ }^{1}$ Department of Pharmacology \& Toxicology, National Institute of Pharmaceutical Education and Research, Guwahati, \\ Assam, India
}

\section{ARTICLE HISTORY}

Received: 29-04-2021

Revised: 30-04-2021

Accepted: 01-05-2021

Online: 02-05-2021

\section{KEYWORDS}

p53

Cancer

Mutations

Clinical studies

\section{ABSTRACT}

p53 is a widely studied oncogene to date. In most cancer types, p53 is highly mutated and considered a potential target for cancer; however, there is no clinically valid candidate in the market that targets p53. There is still confusion about whether p53 promotes cancer? Or not as of now, it is believed that it is a tumor suppressor gene. A lot of studies have been done, and many scientists doubt its functionality. This mini-review will discuss some recent findings on the p53 mutations, their association with cancer, and with some insight on p53 isomers.

\section{Introduction}

p53 is a tumor suppressor gene that functions as a transcription factor with major involvement in DNA repair management, apoptosis, control of cell division, and responses towards cell stresses. Besides, p53 causes the arrest of cell growth and alters cellular aging [1]. TP53 is located at $17 p 13$ locus, which encodes a long protein having 393amino acids. The binding of p53 protein to DNA

\section{*Address for correspondence \\ Department of Pharmacology \& Toxicology, National Institute of Pharmaceutical Education and Research Guwahati, Assam, India. \\ Email: junaidpsst@gmail.com}

DOI: http://dx.doi.org/10.55006/biolsciences.2021.1108 Published by IR Research Publication; Malik JA et al (C) 2021 by Biological Sciences is licensed under CC BY 4.0 (c) activates another gene to translate the protein $\mathrm{p} 21$. p21 crosstalk with CDK2. This CDK2-p21- complex stops the movement of CDK2 to the next level of cell division and prevents unwanted cell growth. This function of p53 describes it as "the guardian of the genome [2]. TP53 is the most commonly altered gene reported in cancer human subjects [3]. In the case of sporadic cancers, the p53 mutations are associated with adverse prognosis [3]. The mutations lead to LOF in p53, which is the main outcome and results in the normal cell transformation to the cancer cell and prevents cells from undergoing apoptosis and senescence. The most frequently associated mutations include missense which leads to amino acid substitution in the DBD. The mutant p53 formed has a different structure from the wild type. It is predicted that it reconstructs the cancer cell transcriptome and proteome because of its crosstalk with many key regulators, proteins, enzymes [4][5]. The cancer cells take full advantage of the new p53 functionality and structure for proliferative potential and survival. The surprising fact is that the mutated p53 becomes 
Abbreviations: PRD: Proline-rich domain; DBD: DNA-binding domain; OD: Oligomerization domain; NLS: Nuclear localization signal; CTD: C terminal domain; TAD: Transactivation domain; AA: Amino acid; CDK2: Cell division stimulating protein; GOF: Gain of function; LOF: Loss of function; AAR: Amino acid residues; DBR: DNA binding region; UCS: Uterine carcinosarcoma; OSCC: Ovarian serous cystadenocarcinoma; VEGFR: Vesicular endothelial growth factor; STAT: Signal transducer activation of transcription; TSGs: Tumour-suppressor-genes; TCGA: The Cancer Genome Atlas; DBD: DNA-binding domain

more stable and gets accumulated inside cells [6], causing the uncontrollable proliferation of cells, leading to malignant tumors.

\section{Mutations}

The p53 mutations are missense, mostly the DBD, which is very unlikely in other tumor suppressor genes. In the DNB, eight amino-acid substitutions are reported in about $27 \%$ mutant p53 proteins as showed by a variety of cancers clinically, as described in Figure 1. It was reported by Ishioka et al. that there were single amino-acid substitutions when they checked the functionality of about nearly domain's apparent fragility, which AA substitutions can impair at various places (Table 1) [8].

\section{The disfunction of p53 Isomers}

There are three members in the p53 family, which are TP53, TP63, and TP73. The increased complexity of this network as the two p53 isoforms (TP63 \& TP73) may also take part in the oncogenesis [8]. The isoforms TP63 \& TP73 are expressed in many forms due to different applications of promoters for alternative splicing and transcription [8]. Long isoforms of p73 or p63 can also transactivate the genes in which p53 can transactivate for induction

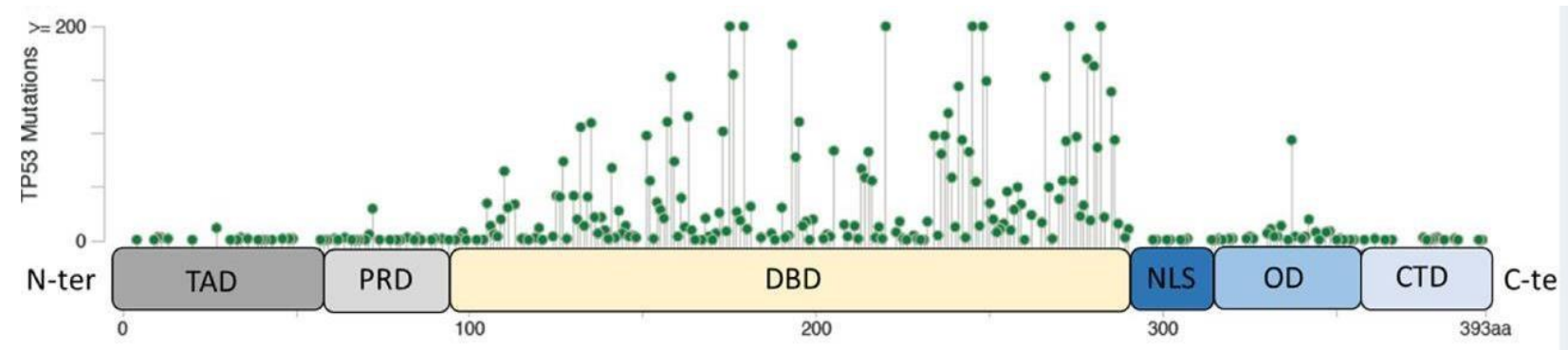

Figure 1. Organization of domains of P53 protein and TP53 missense mutations taken from TCGA. Green dots indicate missense mutations concerning the position of amino-acids and mutation frequency within the amino-acid positions $[1,7]$. The most frequent mutations are these missense mutations in every domain of p53protein, leading to amino acid substitutions and results in LOF and GOF mutation, which ultimately results in cancer transformation

2000 different p53 proteins in yeast [1]. Based on the similar work did by other scientists led to the classification of TP53 gene mutations as described in Table 1. The well-built choice to keep up the expressed full-length p53 proteins in the tumors is extremely advocative for its role in transformation, including dominant negative and gain of function mutations [8]. The ninety-percent p53 mutations are localized in the central domains-100-300 AAR, and most of the p53 proteins are affected by these modifications [8]. The modifications in the p53 are usually due to missense mutations [8] and because of the exposure to mutant elements, there is a high rate of substitutions. The dispersal of p53 mutations is uncommon amid all cancer genes like the tumor suppressor genes and oncogenes. All but seven residues of the 393-AARs of the p53 protein have been the target of at least one mutation in human cancers \& in the core domain that holds the DBR, and each AAR has been detected to be at least five times mutated in individual tumors \& up-to forty thousand times for hotspot mutants. The extensive dispersal of p53 mutations is caused by the core of apoptosis; however, the short isoforms have opposite functionality via dominant-negative mechanistic approaches [8]. There is complex crosstalk between TP73, TP63, and TP53. One reason is their cooperative activity for the induction of apoptosis. The mutations in the p53 can lead to unfolded protein structures that can ligate specifically to p73/p63 and disrupts their activity [8].

\section{The mutations and Cancer}

The p53 mutations were identified in tumors, and germline mutations were described in Li-Fraumeni syndrome, a hereditary cancer predisposition disorder [1]. In humans, most of the p53 mutation occurs due to missense mutation. Mutation of p53 can take place at the early as well as late-stage of cancer. The human body can get an early stage mutation of p53 due to the exposer of any carcinogens. It is reported that the p53 functionality is compromised in thirty-three cancer types, and $1 / 3$ of cancers have a mutation rate in the p 53 gene with a percentage of more than $50 \%$ and $30 \%$ in half of the cancers. UCS and OSCC are the two types of 
Table 1. The Heterogeneity of P53 mutants with examples of the corresponding TP53 mutations

\begin{tabular}{|l|l|c|}
\hline Activity & Examples of amino acid Mutations in p53 & References \\
\hline Gain of function & $\begin{array}{l}\text { R175H,G245S,G245S,R248Q,R248W,S241F,R249S,R273C,R273H,C275Y,R280K,D18 } \\
\text { 1G,R282W }\end{array}$ & {$[1]$} \\
\hline Loss of function & $\mathrm{R} 175 \mathrm{H}, \mathrm{G} 245 \mathrm{~S}, \mathrm{R} 248 \mathrm{Q}, \mathrm{R} 248 \mathrm{~W}, \mathrm{~S} 241 \mathrm{~F}, \mathrm{R} 241 \mathrm{~F}, \mathrm{R} 249 \mathrm{~S}, \mathrm{R} 273 \mathrm{C}, \mathrm{R} 273 \mathrm{H}, \mathrm{C} 275 \mathrm{Y}, \mathrm{R} 280 \mathrm{~K}$ \\
\hline Dominant & $\mathrm{R} 175 \mathrm{H}, \mathrm{G} 245 \mathrm{~S}, \mathrm{R} 248 \mathrm{Q}, \mathrm{R} 248 \mathrm{~W}, \mathrm{~S} 241 \mathrm{~F}, \mathrm{R} 2495, \mathrm{R} 273 \mathrm{C}, \mathrm{R} 273 \mathrm{H}, \mathrm{C} 275 \mathrm{HY}, \mathrm{R} 280 \mathrm{~K}$ \\
\hline Altered specificity & $\mathrm{K} 120 \mathrm{R}, \mathrm{S} 121 \mathrm{~F}, \mathrm{~V} 122 \mathrm{~A}, \mathrm{~T} 125 \mathrm{R}, \mathrm{G} 279 \mathrm{E}$ \\
\hline $\begin{array}{l}\text { Wild type like/Super } \\
\text { Transactivating }\end{array}$ & $\mathrm{T} 123 \mathrm{~A}, \mathrm{G} 199 \mathrm{H}, \mathrm{S} 240 \mathrm{~N}, \mathrm{~S} 288 \mathrm{~K}, \mathrm{R} 337 \mathrm{H}, \mathrm{G} 360 \mathrm{~V}$ \\
\hline $\begin{array}{l}\text { Partial } \\
\text { function/temperate } \\
\text { Sensitive }\end{array}$ & $\mathrm{A} 161 \mathrm{~T}, \mathrm{R} 181 \mathrm{~L}, \mathrm{R} 2025, \mathrm{Y} 220 \mathrm{H}, \mathrm{S} 215 \mathrm{C}, \mathrm{D} 228 \mathrm{~V}, \mathrm{~V} 272 \mathrm{~L}, \mathrm{R} 282 \mathrm{~W}$ \\
\hline
\end{tabular}

cancers in which p53 is highly mutated with the percentage of 91.2 and $83 \%$ [9]. The shift of the p53 tumor suppressor gene to the mutated oncogenic p53 is involving a lot of processes like in the case of wild type p53, which is a cell growth regulator, but when mutations encounter, it can lead to loss of function and can trigger oncogenesis [8]

\section{Mutant p53 in cancer metabolism}

In cancer metabolism, the tumor suppressor gene, p53, plays a balance between glycolysis and mitochondrial respiration. In cancerous cells, the overexpression of glucose transporter is found as a normal phenomenon facilitating glucose flux. It has been found that mutant p53 proteins get stabilized and accumulated in plenty amount within cancer cells through different mechanisms and promote metabolic reprogramming as a novel GOF to promote tumorigenesis [10].

\section{Mutant p53 in Cell Cycle}

It has been found that tumor-derived p53 mutants interact with nuclear factor $Y$. This complex promotes DNA synthesis via upregulating cyclin/CDK1 kinase complexes. These mutations mainly occur at the DNA binding domain of the p53 protein, which is responsible for the downstream stimulations. Thus, mutant p53 protein can no longer effectively bind DNA. For that, p21 will not be available to provide a stop signal to the cell division resulting in uncontrollable tumors [11].

\section{Mutant p53 promotes invasion and migration}

p53/p63 or mutant p53/p73 complexes promote invasion and migration. Due to the formation of these complexes, p62 and p73 get inhibited, enhancing the function of STAT3 signaling and VEGFR expression [12].

\section{Clinical studies}

According to TCGA database out of 4796 clinical cancer cases were affected by about 1423 p53 mutations in 41 projects [7]. The p53 mutations are reported in almost every cancer type with a percentage range from thirty-eight to fifty percent like in esophageal cancer, head cancer, neck cancer, lung cancer, larynx cancer, colorectal cancer and it is five percent in sarcoma, melanoma, cervical cancer, and primary leukemia [13]. The mutation frequency of p53 gets increased in the advanced cancers with unpredictable aggressive behaviors like that of triple negative breast cancer. The polymorphisms have also been identified in the human cancers and its reported that around eighty p53 polymorphisms have been detected are located in the outside splice sites and in the intron regions or at the noncoding regions. In case of smokers suffering from lung cancer about 623 genes were screened from 188 clinical sample which reported that there was higher mutation rate and increased level of guanine to thymidine transversions as compared to non-smokers [14].

\section{Conclusion}

The oncogenic p53 mutations are among the most regularly expressed protein variants in cancers of humans. The distinctive characteristic of p53 is its mode of inactivation compared to other TSGs. It has been reported that more than eighty percent of mutations at the germline and somatic can result in the production of stable mutant $\mathrm{p} 53$-proteins that pile up in the nucleus of the cancer cells for their survival. We can target these mutant $\mathrm{p} 53$ proteins for a better prognosis, but it is challenging, or the drugs can be designed to deplete the mutant p53 from the cells. The mutations in the p53 don't only abolish the tumor suppressor functionality. Still, it is also leading to pro-oncogenic activities, but how the gain of function activities is being achieved is a big question; it requires extensive findings to develop 
strategies to reverse the wild type functionality rather than targeting the mutated p53.

\section{Acknowledgments}

I want to acknowledge the National Institute of Pharmaceutical Education and Research (NIPER), Guwahati, for fellowship.

\section{Conflict of interest}

None

\section{Funding}

None

\section{References}

1. P. Monti, P. Menichini, A. Speciale, G. Cutrona, F. Fais, E. Taiana, A. Neri, R. Bomben, M. Gentile, V. Gattei, M. Ferrarini, F. Morabito, G. Fronza, Heterogeneity of TP53 Mutations and P53 Protein Residual Function in Cancer: Does It Matter?, Frontiers in Oncology. 10 (2020) 2313. https://doi.org/10.3389/fonc.2020.593383.

2. D.E. Moulder, D. Hatoum, E. Tay, Y. Lin, E.M. McGowan, The roles of p53 in mitochondrial dynamics and cancer metabolism: The pendulum between survival and death in breast cancer?, Cancers. $10 \quad$ (2018). https://doi.org/10.3390/cancers10060189.

3. F. Mantovani, L. Collavin, G. del Sal, Mutant p53 as a guardian of the cancer cell, Cell Death and Differentiation. $26 \quad$ (2019) 199-212. https://doi.org/10.1038/s41418-018-0246-9.

4. F. Mantovani, D. Walerych, G. del Sal, Targeting mutant p53 in cancer: a long road to precision therapy, FEBS Journal. 284 (2017) 837-850. https://doi.org/10.1111/febs.13948.

5. M.P. Kim, G. Lozano, Mutant p53 partners in crime, Cell Death and Differentiation. 25 (2018) 161-168. https://doi.org/10.1038/cdd.2017.185.

6. F. Mantovani, L. Collavin, G. del Sal, Mutant p53 as a guardian of the cancer cell, Cell Death and Differentiation. $26 \quad$ (2019) 199-212. https://doi.org/10.1038/s41418-018-0246-9.

7. Genes, (n.d.). https://portal.gdc.cancer.gov/genes/ENSG0000 0141510 (accessed May 1, 2021).
8. T. Soussi, K.G. Wiman, TP53: An oncogene in disguise, Cell Death and Differentiation. 22 (2015) 1239-1249. https://doi.org/10.1038/cdd.2015.53.

9. X. Wang, Q. Sun, TP53 mutations, expression and interaction networks in human cancers, Oncotarget. 8 (2017) 624-643. https://doi.org/10.18632/oncotarget.13483.

10.J. Liu, C. Zhang, W. Hu, Z. Feng, Tumor suppressor p53 and metabolism, Journal of Molecular Cell Biology. 11 (2019) 284-292. https://doi.org/10.1093/jmcb/mjy070.

11.X. Yue, Y. Zhao, Y. Xu, M. Zheng, Z. Feng, W. Hu, Mutant p53 in Cancer: Accumulation, Gain-ofFunction, and Therapy, Journal of Molecular Biology. 429 (2017) 1595-1606. https://doi.org/10.1016/j.jmb.2017.03.030.

12.C. Pitolli, Y. Wang, M. Mancini, Y. Shi, G. Melino, I. Amelio, Do mutations turn p53 into an oncogene?, International Journal of Molecular Sciences. 20 (2019) https://doi.org/10.3390/ijms20246241.

13.M. Olivier, M. Hollstein, P. Hainaut, TP53 mutations in human cancers: origins, consequences, and clinical use., Cold Spring Harbor Perspectives in Biology. 2 (2010). https://doi.org/10.1101/cshperspect.a001008.

14.L. Ding, G. Getz, D.A. Wheeler, E.R. Mardis, M.D. McLellan, K. Cibulskis, C. Sougnez, et al., Somatic mutations affect key pathways in lung adenocarcinoma, Nature. 455 (2008) 1069-1075. https://doi.org/10.1038/nature07423. 